\title{
Hierarchical fragmentation and collapse signatures in a high-mass starless region $\star, \star \star$
}

\author{
H. Beuther ${ }^{1}$, Th. Henning ${ }^{1}$, H. Linz ${ }^{1}$, S. Feng ${ }^{1}$, S. E. Ragan² ${ }^{2}$ R. J. Smith ${ }^{3}$, S. Bihr ${ }^{1}$, T. Sakai ${ }^{4}$, and R. Kuiper ${ }^{5}$ \\ 1 Max-Planck Institute for Astronomy, Königstuhl 17, 69117 Heidelberg, Germany \\ e-mail: name@mpia.de \\ 2 University of Leeds, Leeds, LS2 9JT, UK \\ 3 Jodrell Bank Centre for Astrophysics, School of Physics and Astronomy, The University of Manchester, Oxford Road, Manchester, \\ M13 9PL, UK \\ ${ }^{4}$ Graduate School of Informatics and Engineering, The University of Electro-Communications, Chofu, 182-8585 Tokyo, Japan \\ 5 Institute of Astronomy and Astrophysics, University of Tübingen, Auf der Morgenstelle 10, 72076 Tübingen, Germany
}

Received 16 June 2015 / Accepted 27 July 2015

\begin{abstract}
Aims. We study the fragmentation and collapse properties of the dense gas during the onset of high-mass star formation. Methods. We observed the massive $\left(\sim 800 M_{\odot}\right)$ starless gas clump IRDC 18310-4 with the Plateau de Bure Interferometer (PdBI) at subarcsecond resolution in the $1.07 \mathrm{~mm}$ continuum and $\mathrm{N}_{2} \mathrm{H}^{+}(3-2)$ line emission.

Results. Zooming from a single-dish low-resolution map to previous $3 \mathrm{~mm}$ PdBI data, and now the new 1.07 mm continuum observations, the substructures hierarchically fragment on the increasingly smaller spatial scales. While the fragment separations may still be roughly consistent with pure thermal Jeans fragmentation, the derived core masses are almost two orders of magnitude larger than the typical Jeans mass at the given densities and temperatures. However, the data can be reconciled with models using non-homogeneous initial density structures, turbulence, and/or magnetic fields. While most subcores remain (far-)infrared dark even at $70 \mu \mathrm{m}$, we identify weak $70 \mu \mathrm{m}$ emission toward one core with a comparably low luminosity of $\sim 16 L_{\odot}$, supporting the notion of the general youth of the region. The spectral line data always exhibit multiple spectral components toward each core with comparably small line widths for the individual components (in the 0.3 to $1.0 \mathrm{~km} \mathrm{~s}^{-1}$ regime). Based on single-dish $\mathrm{C}^{18} \mathrm{O}(2-1)$ data we estimate a low virial-to-gas-mass ratio $\leq 0.25$. We propose that the likely origin of these spectral properties may be the global collapse of the original gas clump that results in multiple spectral components along each line of sight. Even within this dynamic picture the individual collapsing gas cores appear to have very low levels of internal turbulence.
\end{abstract}

Key words. stars: formation - stars: early-type - techniques: spectroscopic - stars: individual: IRDC 18310 - ISM: clouds ISM: kinematics and dynamics

\section{Introduction}

Independent of the various formation scenarios for high-mass stars that are discussed extensively in the literature (e.g., Zinnecker \& Yorke 2007; Beuther et al. 2007; Tan et al. 2014), the initial conditions required to allow high-mass star formation at all are still poorly characterized. The initial debate even ranged around the question whether high-mass starless gas clumps should exist at all, or whether the collapse of massive gas clumps starts immediately without any clear starless phase in the high-mass regime (e.g., Motte et al. 2007). Recent studies indicate that the time span during which massive gas clumps exist without embedded star formation is relatively short (on the order of $50000 \mathrm{yr}$ ); nevertheless, high-mass starless gas clumps do exist (e.g., Russeil et al. 2010; Tackenberg et al. 2012; Csengeri et al. 2014). Several questions are currently being considered in this field: are high-mass gas clumps dominated by a single fragment or do we witness strong fragmentation during the earliest

\footnotetext{
* Based on observations carried out with the IRAM Plateau de Bure Interferometer. IRAM is supported by INSU/CNRS (France), MPG (Germany) and IGN (Spain).

$\star \star$ The continuum and spectral line data are only available at the CDS via anonymous ftp to cdsarc.u-strasbg. fr (130.79.128.5) or via http://cdsarc.u-strasbg.fr/viz-bin/qcat?]/A+A/581/A119
}

evolutionary stages (e.g., Bontemps et al. 2010; Zhang et al. 2015)? What are the kinematic properties of the gas (Dobbs et al. 2014)? Are the clumps sub- or super-virial (Tan et al. 2014)? Do we see streaming motions indicative of turbulent flows (e.g., Bergin et al. 2004; Vázquez-Semadeni et al. 2006; Heitsch et al. 2008; Banerjee et al. 2009; Motte et al. 2014; Dobbs et al. 2014)?

To address such questions, one needs to investigate the earliest evolutionary stages prior to the existence of embedded heating and outflow sources that could quickly destroy any signatures of the early kinematic and fragmentation properties. Furthermore, high spatial resolution is mandatory to resolve the important substructures at typical distances of several kpc. To get an idea of the important scales, we can estimate typical Jeans lengths for high-mass star-forming regions; for example, average densities $\rho$ of high-mass star-forming gas clumps on $\sim 0.5 \mathrm{pc}$ scale in the $10^{5} \mathrm{~cm}^{-3}$ density regime at low temperatures of $15 \mathrm{~K}$ result in typical Jeans fragmentation scales of $\sim 10000 \mathrm{AU}$. Going to smaller spatial scales $(\leq 0.1 \mathrm{pc})$, the embedded cores have higher densities in the $10^{6} \mathrm{~cm}^{-3}$ regime that result in much smaller Jeans fragmentation scales on the order of 4000 AU (e.g., Beuther et al. 2013b).

Our target region IRDC $18310-4$ is a $70 \mu \mathrm{m}$ dark high-mass starless region at a distance of $4.9 \mathrm{kpc}$ that was previously observed with the PdBI in the $\mathrm{CD}$ configuration in the $3 \mathrm{~mm}$ 
continuum and the $\mathrm{N}_{2} \mathrm{H}^{+}(1-0)$ emission with a spatial resolution of 4.3" $\times 3.0^{\prime \prime}$ (Beuther et al. 2013b). We identified fragmentation and core formation of the still starless gas clump, and approximate that separations between the separate cores are on the order of $25000 \mathrm{AU}$. Fragmentation of these cores could not be resolved by the previous observations. The simultaneously observed $\mathrm{N}_{2} \mathrm{H}^{+}(1-0)$ data exhibit at least two velocity components. Such multi-velocity-components are the expected signatures of collapsing and fragmenting gas-clumps (Smith et al. 2013).

In a hierarchically structured star-forming region, the cores are the supposed entities where further fragmentation should take place and which likely contain bound multiple systems. Studying this region now at $1.07 \mathrm{~mm}$ in the dust continuum and $\mathrm{N}_{2} \mathrm{H}^{+}(3-2)$ at subarcsecond resolution reveals the hierarchical fragmentation and kinematic properties of the densest cores at the onset of high-mass star formation.

\section{Observations}

\subsection{Plateau de Bure Interferometer}

The target region IRDC 18310-4 was observed with the Plateau de Bure Interferometer at $1.07 \mathrm{~mm}$ wavelength in the B and C configuration in four tracks in March and November 2013. The projected baselines extended to approximately $420 \mathrm{~m}$. The $1 \mathrm{~mm}$ receivers were tuned to $279.512 \mathrm{GHz}$ in the lower sideband. At the given wavelength, the full width half maximum (FWHM) of the primary beam is approximately $18^{\prime \prime}$, and we used a small two-field mosaic to cover our region of interest. Phase and amplitude calibration was conducted with regular observations of the quasars 2013+370 and 1749+096. The absolute flux level was calibrated with MWC349 and bandpass calibration was done with $3 \mathrm{C} 84$. We estimate the final flux accuracy to be correct to within $\sim 15 \%$. The phase reference center is RA (J2000.0) 18:33:39.532 and Dec (J2000.0) -08:21:09.60, and the velocity of rest $v_{\text {lsr }}$ is $\sim 86.5 \mathrm{~km} \mathrm{~s}^{-1}$. While the channel spacing of the correlator was $0.084 \mathrm{~km} \mathrm{~s}^{-1}$, to increase the signal-to-noise ratio $(\mathrm{S} / \mathrm{N})$ we imaged the data with $0.2 \mathrm{~km} \mathrm{~s}^{-1}$ spectral resolution. For the continuum and line data we used different weighting systems between natural and uniform weighting to improve the $\mathrm{S} / \mathrm{N}$ in particular for the line data. The resulting synthesized beam for the continuum and $\mathrm{N}_{2} \mathrm{H}^{+}(3-2)$ data were $0.6^{\prime \prime} \times 0.49^{\prime \prime}$ (with a position angle of $11^{\circ}$ ) and $1.13^{\prime \prime} \times 0.5^{\prime \prime}$ (with a position angle of $13^{\circ}$ ), respectively. The corresponding $1 \sigma \mathrm{rms}$ for the two datasets is $\sim 0.6 \mathrm{mJy} \mathrm{beam}^{-1}$ and $\sim 15 \mathrm{mJy}^{-1}$ beam $^{-1}$ measured in a line-free channel of $0.2 \mathrm{~km} \mathrm{~s}^{-1}$ for the line data.

\subsection{Herschel/PACS}

The Herschel data were already presented in Beuther et al. (2013a). However, they were fully re-calibrated for this analysis with particular emphasis on applying the most recent pointing and astrometry solutions. The region containing IRDC 18310-4 was observed with the Herschel spacecraft (Pilbratt et al. 2010) within the Key Project EPoS (Ragan et al. 2012). The related observations utilizing the bolometer cameras of PACS (Poglitsch et al. 2010) took place on April 19, 2011 (operational day 705), and were contained in the observational IDs 1342219060-63. The PACS prime mode was employed with a nominal scanning velocity of $20^{\prime \prime} \mathrm{s}^{-1}$. Data were taken in all three PACS filters $(70,100$, and $160 \mu \mathrm{m})$ and resulted in maps with roughly 10 arcmin field of view. We re-reduced the existing Herschel archive data, starting from the Level 1 data. We retrieved the data from the previous bulk processing with the HIPE 12.1 software version, currently contained in the HSA. We included the newly developed data reduction step calcAttitude: a correction of the frames pointing product based on the Herschel gyroscope housekeeping (Sánchez-Portal et al. 2014). This often improves the absolute pointing accuracy and mitigates the pointing jitter effect on individual frames. We used these corrected frames and performed the individual detector pixel distortion correction, the de-striping, the removal of $1 / \mathrm{f}$ noise, and the final map projection using Scanamorphos (Roussel 2013) version 24, employing the "galactic" option. The resulting FWHM of the point spread function (PSF) at 70, 100, and $160 \mu \mathrm{m}$ are 5.6", 6.8", and 11.3", respectively.

\section{Results}

\subsection{Millimeter continuum emission}

Figure 1 presents a compilation of the $70 \mu \mathrm{m}$ and $1.2 \mathrm{~mm}$ singledish data (Ragan et al. 2012; Beuther et al. 2002), the previous $3.2 \mathrm{~mm}$ PdBI continuum observations (Beuther et al. 2013b), and our new $1.07 \mathrm{~mm}$ PdBI continuum maps. The three datasets cover a broad range of resolution elements from $11^{\prime \prime}$ in the MAMBO $1.2 \mathrm{~mm}$ data, to $4.3^{\prime \prime} \times 3.0^{\prime \prime}$ in the $3.2 \mathrm{~mm}$ PdBI data, to $0.6^{\prime \prime} \times 0.49^{\prime \prime}$ in our new $1.07 \mathrm{~mm}$ observations. At the given distance of $4.9 \mathrm{kpc}$, this corresponds to linear resolution elements of $\sim 54000 \mathrm{AU}, \sim 18000 \mathrm{AU}$, and $\sim 2700 \mathrm{AU}$, respectively. With the different resolution elements, we can clearly identify hierarchical fragmentation on the different scales of our observations: the large-scale single-dish gas clump with a total mass of $\sim 800 M_{\odot}$ fragments into four cores with masses between 18 and $36 M_{\odot}$ (Beuther et al. 2013b), and these cores again fragment into smaller substructures in our new $1.07 \mathrm{~mm}$ continuum data. For our following analysis, we only consider $1.07 \mathrm{~mm}$ sources that are detected at $a>4 \sigma$ level. We can estimate the masses and column densities of these smallest-scale structures with similar assumptions as taken in Beuther et al. (2013b), namely optically thin dust emission at a low temperature of $15 \mathrm{~K}$ (see also Sect. 3.2), with a gas-to-dust ratio of 150 (Draine 2011) and dust properties discussed in Ossenkopf \& Henning (1994) for thin ice mantles at densities of $10^{5} \mathrm{~cm}^{3}\left(\kappa_{1.07 \mathrm{~mm}} \sim 0.95 \mathrm{~cm}^{2} \mathrm{~g}^{-1}\right)$. Main uncertainties for the mass and column density estimates are the applied dust model and the assumed temperature, which leads us to estimate an accuracy within a factor 2 for these parameters. In this way, we estimate the masses of the smallest-scale substructures to values between 2.2 and $19.3 M_{\odot}$ (Table 1 ). The difference between the sum of the core masses and the large-scale single-dish mass of $\sim 800 M_{\odot}$ is mainly caused by the spatial filtering of the interferometer that traces only the densest inner cores and no longer the envelope. The peak column densities are very high in the regime of $10^{24} \mathrm{~cm}^{-2}$, corresponding to visual extinctions of $\sim 1000 \mathrm{mag}$.

In addition to the masses and column densities, the data allow us to estimate the fragmentation properties of the subsources, and we find projected separations between the newly identified structures between 2600 and 12000 AU (Table 2). Since the absolute peak positions can be determined to high accuracy $\left(\frac{\phi}{S / N}\right.$ with the resolution $\phi$ and the $\mathrm{S} / \mathrm{N}$; e.g., Reid et al. 1988), and the intereferometric positional accuracy depends mainly on the position of the quasar used and its gain calibration solution (within $0.1^{\prime \prime}$ ), the projected separation is estimated to be accurate within $0.1^{\prime \prime}$ or 490 AU. A fragmentation discussion is presented in Sect. 4.1. 
H. Beuther et al.: Hierarchical fragmentation and collapse signatures

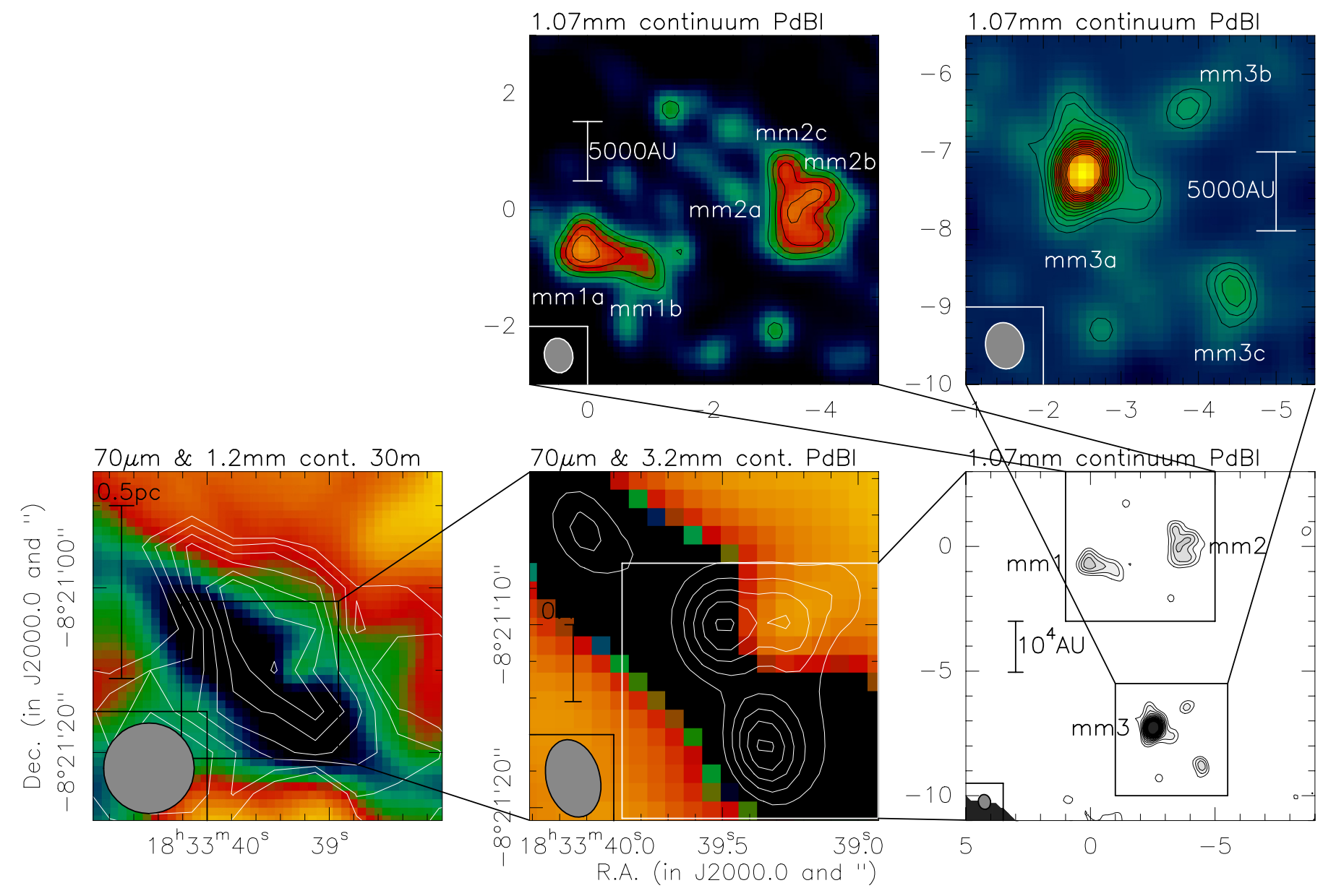

Fig. 1. Compilation of the continuum images in IRDC 18310-4. The bottom left and middle panels show in color the Herschel $70 \mu \mathrm{m}$ image with a stretch going dark for low values (Beuther et al. 2013b, linear and log stretch for clarity, respectively). The white contours in the left panel show the $1.2 \mathrm{~mm}$ MAMBO continuum observations starting from $4 \sigma$ and continuing in $1 \sigma$ steps with a $1 \sigma$ value of $13 \mathrm{mJy} \mathrm{beam}^{-1}$. The contours in the bottom middle panel present the $3 \mathrm{~mm}$ continuum data from Beuther et al. (2013b) starting from $3 \sigma$ and continuing in $2 \sigma$ steps with a $1 \sigma$ value of $0.08 \mathrm{mJy}_{\text {beam }}{ }^{-1}$. The bottom right panel then shows the new $1.07 \mathrm{~mm}$ continuum observations starting from $3 \sigma$ and continuing in $1 \sigma$ steps with a $1 \sigma$ value of $0.6 \mathrm{mJy}_{\text {beam }}{ }^{-1}$. The two top panels show zooms of the 1.07 continuum data with the same contour levels but a different color stretch to highlight the substructures. Each panel presents scale bars and the corresponding synthesized beams. The $70 \mu \mathrm{m}$ PSF is 5.6".

Table 1. Fluxes, masses, and column densities.

\begin{tabular}{lrrrr}
\hline \hline & $\begin{array}{r}S_{\text {int }} \\
(\mathrm{mJy})\end{array}$ & $\begin{array}{r}S_{\text {peak }} \\
\left(\frac{\mathrm{mJy}}{\text { beam }}\right)\end{array}$ & $\begin{array}{r}M \\
\left(M_{\odot}\right)\end{array}$ & $\begin{array}{r}N_{\mathrm{H}_{2}} \\
\left(10^{24} \mathrm{~cm}^{-2}\right)\end{array}$ \\
\hline $\mathrm{mm} 1$ & $12.0^{1}$ & & $9.7^{1}$ & \\
$\mathrm{~mm} 1 \mathrm{a}$ & 8.2 & 4.1 & 6.6 & 1.1 \\
$\mathrm{~mm} 1 \mathrm{~b}$ & 3.8 & 2.9 & 3.1 & 0.8 \\
$\mathrm{~mm} 2$ & $17.0^{2}$ & & $13.7^{2}$ & \\
$\mathrm{~mm} 2 \mathrm{a}$ & 4.5 & 3.8 & 3.6 & 1.0 \\
$\mathrm{~mm} 2 \mathrm{~b}$ & 4.7 & 3.8 & 3.8 & 1.0 \\
$\mathrm{~mm} 2 \mathrm{c}$ & 3.3 & 3.3 & 2.7 & 0.9 \\
$\mathrm{~mm} 3 \mathrm{a}$ & 24.0 & 14.0 & 19.3 & 3.8 \\
$\mathrm{~mm} 3 \mathrm{~b}$ & 2.7 & 2.7 & 2.2 & 0.7 \\
$\mathrm{~mm} 3 \mathrm{c}$ & 3.5 & 3.5 & 2.8 & 0.9 \\
\hline
\end{tabular}

Notes. Uncertainties for $S_{\text {int }}$ and $S_{\text {peak }}$ are $\approx 15 \%$ (Sect. 2). Uncertainties for $M$ and $N_{\mathrm{H}_{2}}$ are approximately a factor of 2 (Sect. 3.1). ${ }^{(1)}$ Integrated over $\mathrm{mm} 1$ and mm1a. ${ }^{(2)}$ Integrated over $\mathrm{mm} 2 \mathrm{a}, \mathrm{mm} 2 \mathrm{~b}$, and $\mathrm{mm} 2 \mathrm{c}$.

\subsection{Far-infrared continuum emission}

Figure 2 presents a comparison of the Herschel/PACS 70, 100, and $160 \mu \mathrm{m}$ data with the $3.2 \mathrm{~mm}$ continuum data from the PdBI. At $70 \mu \mathrm{m}$, large areas of the central region containing the
Table 2. Projected nearest neighbor separations.

\begin{tabular}{lrr}
\hline \hline & $\left({ }^{\prime \prime}\right)$ & $(\mathrm{AU})$ \\
\hline 3 mm data & & \\
mm1-mm2 & 2.9 & 14200 \\
mm1-mm3 & 7.1 & 34900 \\
\hline $1 \mathrm{~mm}$ data & & \\
mm1a-mm1b & 0.9 & 4400 \\
mm2a-mm2b & 0.5 & 2600 \\
mm2a-mm2c & 0.7 & 3500 \\
mm2b-mm2c & 1.1 & 5500 \\
mm3a-mm3b & 1.5 & 7600 \\
mm3a-mm3c & 2.5 & 12000 \\
mm3b-mm3c & 2.4 & 11900 \\
\hline
\end{tabular}

Notes. Uncertainties are $\approx 0.1^{\prime \prime}$ or $\approx 490 \mathrm{AU}$.

millimeter cores still appear as a dark extinction silhouette in front of the extended $70 \mu \mathrm{m}$ emission of the hosting molecular cloud (Figs. 1 and 2). Although the extinction contrast decreases at $100 \mu \mathrm{m}$, we still perceive the central parts as an infrared dark cloud (IRDC). At $160 \mu \mathrm{m}$, the extended emission in the surroundings does not dominate as much, and emission structures emerge from the inside of the IRDC. 


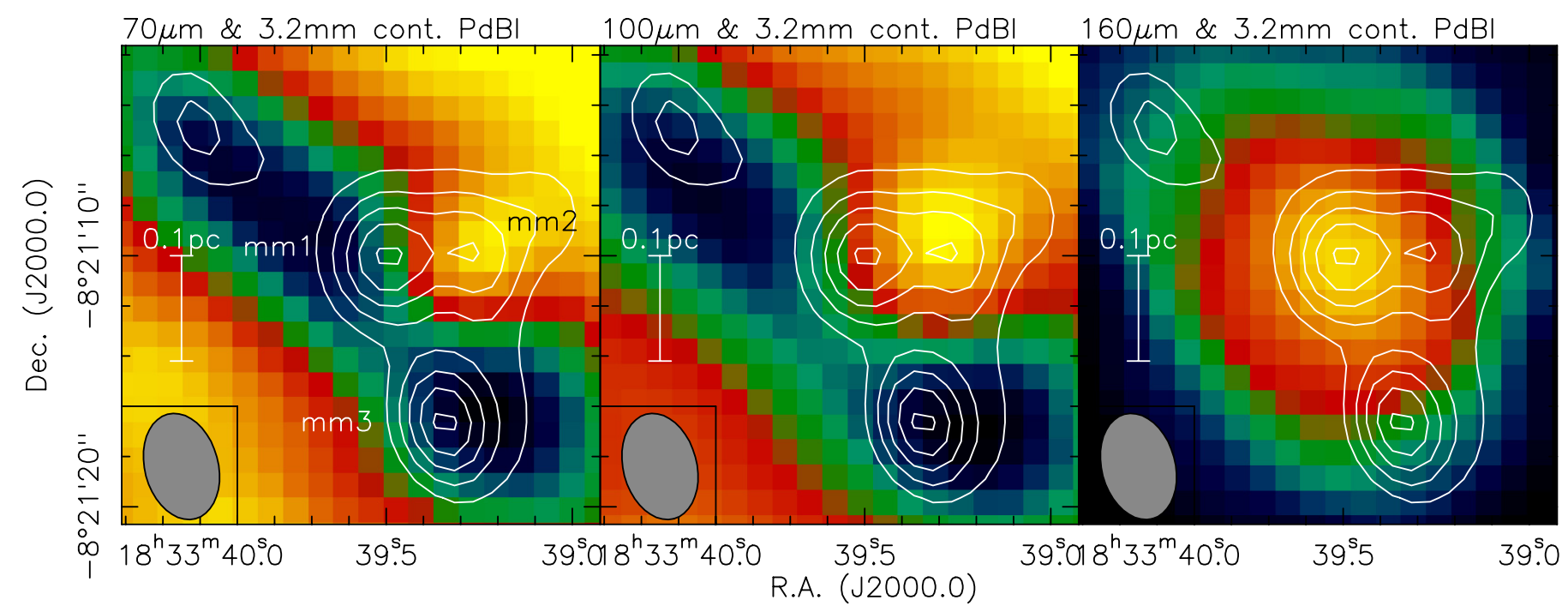

Fig. 2. Re-calibrated Herschel/PACS images at 70, 100, and $160 \mu \mathrm{m}$ (from left to right). The scaling in the three panels is from -3.8 to 4.0 , from 5.0 to 17.6 , and from 33 to $45 \mathrm{mJy}_{\text {pixel }}{ }^{-1}$, respectively. The $3.2 \mathrm{~mm}$ continuum contours are from $3 \sigma$ and continue in $2 \sigma$ steps with a $1 \sigma$ value of $0.08 \mathrm{mJy}_{\text {beam }}{ }^{-1}$. A scale bar and the $3.2 \mathrm{~mm}$ synthesized beam are shown in each panel, and the three main sources are labeled in the bottom left panel. The 70, 100, and $160 \mu \mathrm{m}$ PSFs are $5.6^{\prime \prime}, 6.8^{\prime \prime}$, and $11.3^{\prime \prime}$, respectively.

At the northwestern border of the IRDC silhouette, we notice a faint emission point source at $70 \mu \mathrm{m}$ which also persists at $100 \mu \mathrm{m}$. In the previous data reduction products we used in Ragan et al. (2012) and Beuther et al. (2013a), this source was not as apparent. We attribute the differences to the use of the newest Scanamorphos algorithms and the inclusion of the gyro-correction information (Sect. 2.2) in the current data reduction. In order to determine accurate positions, we referenced the PACS maps with the Spitzer/MIPSGAL (Carey et al. 2009) $24 \mu \mathrm{m}$ data. We did not use individual MIPSGAL maps for reference, but relied on the recently released MIPSGAL $24 \mu \mathrm{m}$ point source catalogue (Gutermuth \& Heyer 2015). Corresponding positions in the PACS maps were determined by employing PSF photometry using IDL/Starfinder (Diolaiti et al. 2000). The necessary adjustments were shifts on the order of 1.1-1.6". For the previous Herschel data products (with uncorrected pointing) we had to use a shift of almost $4^{\prime \prime}$ (Beuther et al. 2013a). This supports predictions that the absolute pointing error of Herschel data can be brought down to a level of $\sim 0.91 \sigma$ if all the recent corrections for the pointing product are applied (Sánchez-Portal et al. 2014). This $70 \mu \mathrm{m}$ point source is thus located very close to $\mathrm{mm} 2$. Interestingly, the compact emission seen at $160 \mu \mathrm{m}$ is shifted toward the center of the IRDC and basically coincides with the mm1 location (Fig. 2). The uncertainties of the peak positions are on the order of $1^{\prime \prime}$ for 70 and $100 \mu \mathrm{m}$, due to the position bootstrapping involved. For the $160 \mu \mathrm{m}$ astrometry, the uncertainty might even be up to $2^{\prime \prime}$, since at this wavelength it is hard to find real point sources, and owing to extended emission and large beam sizes the effective peak position may experience subtle displacements (cf. the comparison of MIPS-70 and PACS-70 $\mu \mathrm{m}$ peaks for the EPoS source UYSO1 reported in Linz et al. 2010). Still, the formal distance between the $70 \mu \mathrm{m}$ point source and the $160 \mu \mathrm{m}$ compact emission peak is more than $4^{\prime \prime}$. Although this is less than the beam of the $70 \mu \mathrm{m}$ image, as mentioned in Sect. 3.1, source peak positions can be determined to much higher accuracy than the nominal spatial resolution $\phi$ (down to $\frac{\phi}{S / N}$ ). Hence, we think that there is a real shift in peak positions when going to longer wavelengths. This can be explained by the combined action of two effects. First, the $160 \mu \mathrm{m}$ data trace colder dust in the deeper interior of the IRDC
Table 3. Far-infrared fluxes of $\mathrm{mm} 2$.

\begin{tabular}{lr}
\hline \hline$\lambda$ & $\begin{array}{r}S \\
(\mu \mathrm{m})\end{array}$ \\
\hline 70 & 90 \\
100 & 357 \\
$160^{1}$ & $\leq 339$ \\
$3230^{2}$ & 0.9 \\
\hline
\end{tabular}

Notes. Flux uncertainties at 70 and $100 \mu \mathrm{m}$ are $\sim 10 \%$. ${ }^{(1)}$ Upper limit from the residual flux toward $\mathrm{mm} 2$ after subtracting the point source centered on mm1. ${ }^{(2)}$ From Beuther et al. (2013a) with an accuracy of $\sim 15 \%$.

than the $70 \mu \mathrm{m}$ wavelength range does. Second, the $160 \mu \mathrm{m}$ beam is around $11^{\prime \prime} 3$. It thus convolves emission from larger areas, and therefore may comprise emission from $\mathrm{mm} 1$ (dominating) and $\mathrm{mm} 2$ (minor contribution), while the finer $70 \mu \mathrm{m}$ beam of 5." 6 can still distinguish between $\mathrm{mm} 2$ (associated with $70 \mu \mathrm{m}$ emission at its northwest side) and $\mathrm{mm} 1$ (without noticeable $70 \mu \mathrm{m}$ emission).

Hence, the PACS data show tentatively that at least object $\mathrm{mm} 2$ may no longer be totally starless, but that star formation processes might have begun at its northwestern border. Whether the $70 \mu \mathrm{m}$ point source is already a recently formed protostar, or just a temperature enhancement created by various processes triggered from within $\mathrm{mm} 2$, is not easy to determine with the presently available data. At $160 \mu \mathrm{m}$, we then see the cold dust emission from the bulk of the core material in the $\mathrm{mm} 1 / 2$ region.

The Herschel data can also be used to get a rough estimate of the luminosity of the $\mathrm{mm} 2$ core. In the 70 and $100 \mu \mathrm{m}$ bands, we were able to derive the far-infrared fluxes toward $\mathrm{mm} 2$ by fitting the PSF to the compact emission source visible in Fig. 2. The uncertainties for the 70 and $100 \mu \mathrm{m}$ flux measurements are $\approx 10 \%$, including $\sim 5 \%$ calibration uncertainty and another $\sim 5 \%$ from the PSF photometry. At $160 \mu \mathrm{m}$, this is more difficult because the emission peaks at mm1. Therefore, in this band we can only derive an upper limit for $\mathrm{mm} 2$ by first fitting a point source to $\mathrm{mm} 1$ and then deriving the $\mathrm{mm} 2$ upper limit from the residual image. The $3 \mathrm{~mm}$ flux density was taken from Beuther et al. (2013a). Fitting a spectral energy distribution to these four 


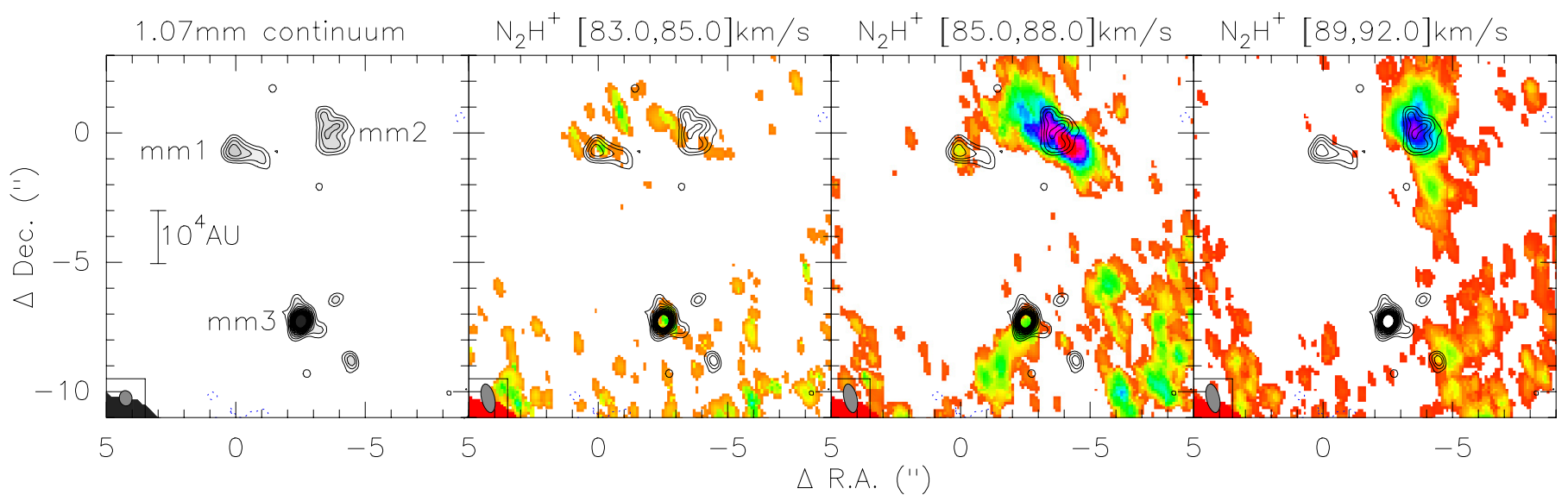

Fig. 3. $\mathrm{N}_{2} \mathrm{H}^{+}(3-2)$ and $1.07 \mathrm{~mm}$ continuum data. The left panel and the contours in the three other panels always show the $1.07 \mathrm{~mm}$ continuum data starting from $3 \sigma$ and continuing in $1 \sigma$ steps with a $1 \sigma$ value of $0.6 \mathrm{mJy} \mathrm{beam}^{-1}$. The color scale in panels $2-4$ present the $\mathrm{N}_{2} \mathrm{H}^{+}(3-2)$ integrated emission over the velocity regimes marked above each panel. The scaling ranges in panels 2 to 4 are $[0,0.15],[0,0.25]$, and $[0,0.4] \mathrm{Jy} \mathrm{km} \mathrm{s}^{-1}$, respectively. The synthesized beam of the continuum and line data is shown each time in the bottom left. A scale bar can be found in the left panel.

data points assuming a modified blackbody function accounting for the wavelength-dependent emissivity of the dust, we get an estimate of the luminosity $L$ and cold dust temperature $T_{\text {dust }}$. For $\mathrm{mm} 2$, this results in $L \sim 16_{-8}^{+14} L_{\odot}$ and $T_{\text {dust }} \sim 15_{-0.5}^{+2.0} \mathrm{~K}$. The error budget in $L$ and $T_{\text {dust }}$ includes the uncertainties of the flux calibration and of the selected dust model. While the dust temperature reflects the overall cold nature of this region, the lower internal luminosity of $\mathrm{mm} 2$ around $16 L_{\odot}$ shows that this region with a large mass reservoir of $\sim 800 M_{\odot}$ (Sect. 3.1) has not yet formed a high-mass star. It should also be noted that the low luminosity cannot be explained well with accretion processes on compact protostars because even then the accretion luminosity is expected to be higher (e.g., Krumholz et al. 2007). Hence, the observed luminosity may likely stem from accretion processes on larger surfaces, re-enforcing the early evolutionary stage at the onset of star formation with active collapse processes.

Looking closer at $\mathrm{mm} 3$, there is no emission at 70 and $100 \mu \mathrm{m}$, and the $160 \mu \mathrm{m}$ image exhibits only a very weak extension from $\mathrm{mm} 1$ in the direction of $\mathrm{mm} 3$. This is in stark contrast to the 3.2 and $1.07 \mathrm{~mm}$ data which exhibit almost the same fluxes for $\mathrm{mm} 1$ and $\mathrm{mm} 3$. This difference is most likely due to even lower temperatures within the $\mathrm{mm} 3$ region compared to $\mathrm{mm} 1$.

\subsection{Spectral line emission}

Figure 3 presents several $\mathrm{N}_{2} \mathrm{H}^{+}(3-2)$ emission maps integrated each time over different velocity regimes. The velocity regimes are selected based on the integrated $\mathrm{N}_{2} \mathrm{H}^{+}(3-2)$ spectrum presented in the bottom panel of Fig. 4. We divide the integrations into a low-velocity regime between 83 and $85 \mathrm{~km} \mathrm{~s}^{-1}$, an intermediate-velocity regime between 85 and $88 \mathrm{~km} \mathrm{~s}^{-1}$, and a high-velocity regime from 89 to $92 \mathrm{~km} \mathrm{~s}^{-1}$. The immediate result of this division is that the different velocities trace different parts of the dense gas. While the low-velocity component is mainly associated with the subsources $\mathrm{mm} 1$ and $\mathrm{mm} 3$, the intermediatevelocity component emits toward all three mm cores, and finally the high-velocity component emits only toward $\mathrm{mm} 2$. Although the $\mathrm{N}_{2} \mathrm{H}^{+}(3-2)$ line has hyperfine structure as well, the satellite lines are comparably weak, and the velocity structure is mainly caused by real structure imposed on the main hyperfine component. Nevertheless, we also fit the spectra taking into account the full hyperfine structure of the line (see below). Independent
Table 4. Hyperfine structure fits toward the mm peaks.

\begin{tabular}{lrr}
\hline \hline & $\begin{array}{r}v_{\text {peak }} \\
\left(\mathrm{km} \mathrm{s}^{-1}\right)\end{array}$ & $\begin{array}{r}\Delta v \\
\left(\mathrm{~km} \mathrm{~s}^{-1}\right)\end{array}$ \\
\hline $\mathrm{mm} 1$ & 83.5 & 0.3 \\
$\mathrm{~mm} 1$ & 84.3 & 0.3 \\
$\mathrm{~mm} 1$ & 86.3 & 0.3 \\
\hline $\mathrm{mm} 2$ & 85.8 & 1.3 \\
$\mathrm{~mm} 2$ & 89.9 & 0.7 \\
$\mathrm{~mm} 2$ & 91.0 & 0.3 \\
\hline $\mathrm{mm} 3$ & 84.4 & 0.6 \\
$\mathrm{~mm} 3$ & 86.2 & 0.9 \\
\hline
\end{tabular}

of this fit, the integrated emission images already show that this high-mass starless clump is far from being a kinematically homogeneous and potentially calm structure, but in contrast, we see a kinematically complex region that may be at the verge of collapse (see also Ragan et al. 2015).

To investigate the kinematics in greater depth, we extracted the spectra toward the three main $\mathrm{mm}$ cores, $\mathrm{mm} 1, \mathrm{~mm} 2$, and $\mathrm{mm} 3$ (see Fig. 4). Again, the multiple velocity components are apparent. To quantify the peak velocities $v_{\text {peak }}$ and FWHM $\Delta v$, we fitted the full $\mathrm{N}_{2} \mathrm{H}^{+}(3-2)$ hyperfine structure to these lines with multiple components. We note that, in contrast to the 1-0 transition, for the 3-2 transition of $\mathrm{N}_{2} \mathrm{H}^{+}$the spectrum is much more strongly dominated by the central line component. From the 29 hyperfine structure components more than $60 \%$ of the relative intensities are within the central 6 components separated by only $\sim 0.06 \mathrm{~km} \mathrm{~s}^{-1}$, whereas the remaining 23 components share the rest of the emission. The resulting fits are shown in Table 4 . We note that the selection of the number of fitted velocity components is partly ambiguous. For example, for $\mathrm{mm} 2$ we fitted a total of three components, but it would also be possible to fit the high-velocity feature in $\mathrm{mm} 2$ with three components alone and have a fourth component around the intermediatevelocity gas. Therefore, these fits do not claim to be the final answer, although they adequately represent the current data. While the multiple components toward individual peaks are interesting in themselves, the derived line widths are also important. They range in FWHM between 0.3 and $1.3 \mathrm{~km} \mathrm{~s}^{-1}$, where the $0.3 \mathrm{~km} \mathrm{~s}^{-1}$ has to be considered as an upper limit because with our velocity resolution of $0.2 \mathrm{~km} \mathrm{~s}^{-1}$ no narrower lines can be resolved. The broad end of the distribution is also most likely an 

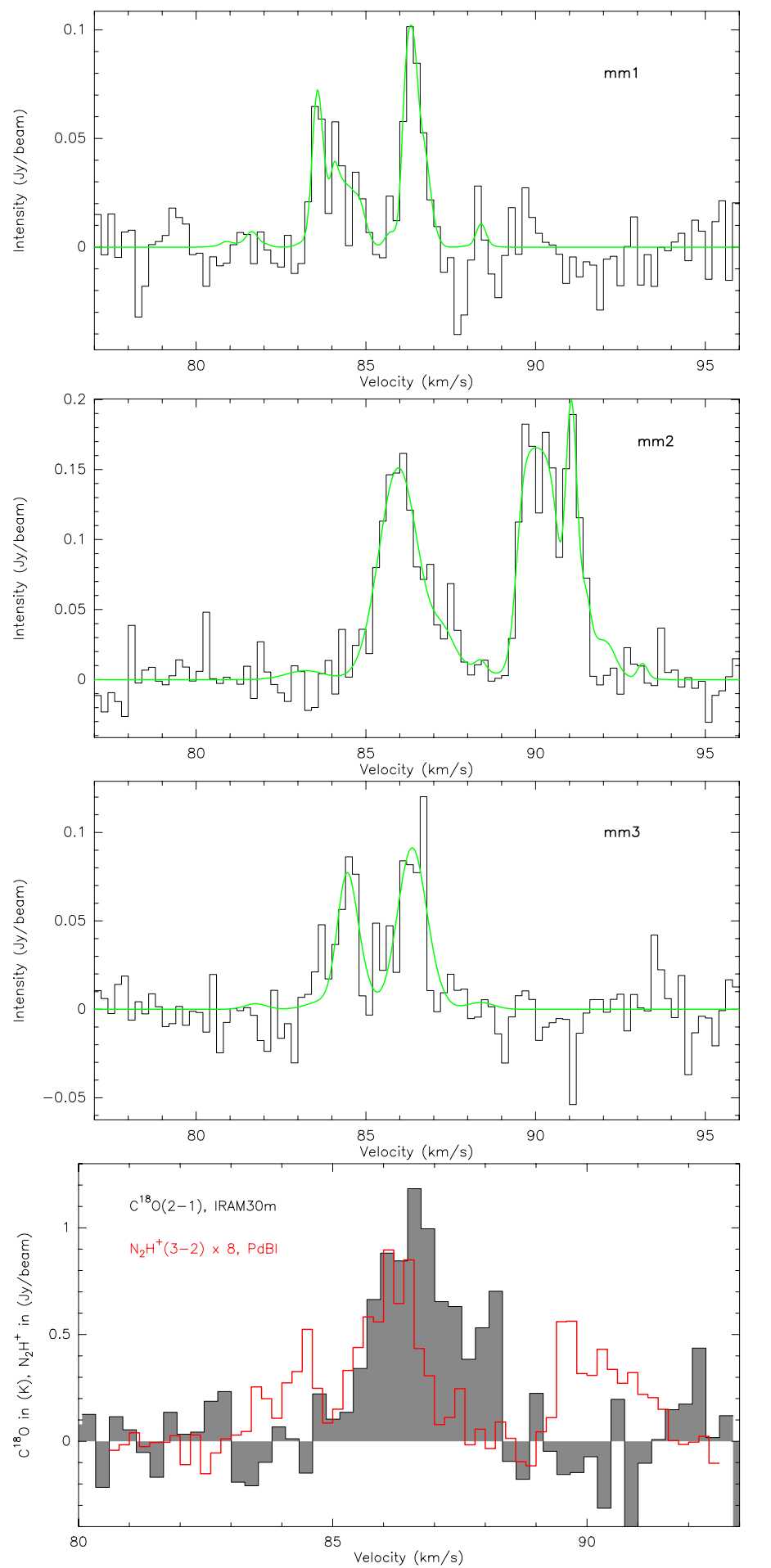

Fig. 4. $\mathrm{N}_{2} \mathrm{H}^{+}(3-2)$ spectra toward the three main peaks mm1, mm2, and $\mathrm{mm} 3$ with the multiple component hyperfine structure fits shown in green. The fit parameters are presented in Table 4 . The bottom spectrum shows a comparison between a single-dish $\mathrm{C}^{18} \mathrm{O}(2-1)$ spectrum extracted toward the main peak in gray and an average of the above three $\mathrm{N}_{2} \mathrm{H}^{+}(3-2)$ spectra (multiplied by 8 ) in red. The units in the bottom spectra are different for both as marked in the side label.

upper limit, however, in this case caused by unresolved underlying spectral multiplicity in the lines. With the thermal line width at $15 \mathrm{~K}$ of $\sim 0.15 \mathrm{~km} \mathrm{~s}^{-1}$, these new data now resolve the spectra into almost thermal lines, just with multiple components.
For comparison, Figure 4 also shows a single-dish $\mathrm{C}^{18} \mathrm{O}(2-1)$ spectrum at $11^{\prime \prime}$ resolution (Ragan et al., priv. comm.) as well as the PdBI $\mathrm{N}_{2} \mathrm{H}^{+}(3-2)$ spectrum integrated over all three $\mathrm{mm}$ continuum cores. While the $\mathrm{C}^{18} \mathrm{O}$ spectrum easily traces the main intermediate-velocity component, although spectrally not well resolved in the subcomponents (a Gaussian two-component fit to the $\mathrm{C}^{18} \mathrm{O}(2-1)$ spectrum results in $\Delta v$ values of $\sim 1.7$ and $\sim 0.3 \mathrm{~km} \mathrm{~s}^{-1}$, a one-component fit has $\Delta v \sim 2.0 \mathrm{~km} \mathrm{~s}^{-1}$ ), the high- and low-velocity components are not seen at all in the $\mathrm{C}^{18} \mathrm{O}(2-1)$ spectrum. There are two likely reasons for this: the lower spatial resolution of the single-dish data and the lower critical density of $\mathrm{C}^{18} \mathrm{O}(2-1)$ compared to $\mathrm{N}_{2} \mathrm{H}^{+}(3-2)$ (by about two orders of magnitude: $\sim 10^{4}$ versus $\left.\sim 10^{6} \mathrm{~cm}^{-3}\right)$. With the new high-resolution observations of this dense gas tracer, we are able to dissect the densest portions of this very young high-mass star-forming region (see Sect. 4.2).

\section{Discussion}

We now have the ability to track the hierarchical fragmentation and global collapse of starless gas clumps capturing smaller and smaller scales.

\subsection{Fragmentation}

Following Beuther et al. (2013b), we can estimate the average densities $\rho$ for the large-scale single-dish data as well as the previous intermediate-scale $3 \mathrm{~mm}$ continuum data, corresponding to the bottom left and bottom middle panels in Fig. 1. The estimated average densities $\rho$ derived from the two datasets are $2.5 \times 10^{5} \mathrm{~cm}^{-3}$ and $1.5 \times 10^{6} \mathrm{~cm}^{-3}$, respectively. In addition, by assuming average temperatures of $15 \mathrm{~K}$, Beuther et al. (2013b) estimated the Jeans length $\lambda_{\text {Jeans }}$ and Jeans mass $M_{\text {Jeans }}$ that predict the expected fragment properties of the corresponding smaller scales in the framework of this isothermal gravitational Jeans fragmentation picture. The $\lambda_{\text {Jeans }}$ and $M_{\text {Jeans }}$ based on the large-scale single-dish data are $10000 \mathrm{AU}$ and $0.37 M_{\odot}$, whereas the corresponding $\lambda_{\text {Jeans }}$ and $M_{\text {Jeans }}$ derived from the intermediate-scale $3 \mathrm{~mm}$ PdBI data are $4000 \mathrm{AU}$ and $0.15 M_{\odot}$ (Beuther et al. 2013b).

If we now compare the predicted length and mass scales with the corresponding observed values on the different scales, we find correspondences as well as differences. Regarding the Jeans length and the observed projected separations, the predicted Jeans length from the single-dish data of $\sim 10000 \mathrm{AU}$ is slightly smaller than the core separation in the PdBI $3.2 \mathrm{~mm}$ data (Table 2), but the values are consistent within a factor of a few. Going to smaller scales, the predicted Jeans length from the $3.2 \mathrm{~mm}$ data of $\sim 4000 \mathrm{AU}$ is roughly consistent with the projected separations we find again at the smaller scales of the $1.07 \mathrm{~mm}$ continuum data (Table 2 ). Hence, from a pure lengthscale argument, the observations of IRDC 18310-4 would be roughly consistent with classical thermal Jeans fragmentation. However, our observations reveal only projected separations, and the real values can be up to a factor of 2 larger.

Does this picture also hold for the Jeans masses? In fact it does not because the predicted Jeans masses on the different scales of 0.37 and $0.15 M_{\odot}$, respectively, are significantly lower than what is found in the $3.2 \mathrm{~mm}$ data (masses for $\mathrm{mm} 1$ to $\mathrm{mm} 3$ between 18 and $36 M_{\odot}$, Beuther et al. 2013b) as well as in our new $1.07 \mathrm{~mm}$ observations (masses between 2.2 and $13.7 M_{\odot}$, Table 1). Hence, while the length scales are roughly consistent between the classical Jeans predictions and the data, the masses deviate by up to two orders of magnitude. We note that these 
observed masses are only lower limits because large fractions of the gas are filtered out.

Similar discrepancies were recently reported by Wang et al. (2014) in their fragmentation study of parts of what is known as the Snake filament. They also found projected separations in their data that correspond reasonably well to the estimated Jeans length, whereas the fragment masses exceeded the Jeans masses by similar margins to the values in our data. They argued that the typical thermal Jeans mass is calculated with the thermal sound speed depending on the temperature of the gas. However, Wang et al. (2014) evaluate a turbulent Jeans mass using the turbulent velocity dispersion of the gas instead of the velocity dispersion based on the thermal sound speed. Since the Jeans mass depends on the velocity dispersion to the third power, even velocity dispersion increases of a factor of a few allow the turbulent Jeans masses to shift by more than an order of magnitude in their regime of observed masses. Hence, Wang et al. (2014) argue that turbulent Jeans fragmentation may explain the observed properties. A first-order inconsistency could arise in this picture when considering the length scales. In the turbulent Jeans scenario, the turbulent Jeans length also has to be adapted according to the turbulent velocity dispersion. Although the velocity dispersion only enters the equation for the Jeans length linearly; nevertheless, it increases the turbulent Jeans length by a factor of a few. And in that picture, the projected separations of their cores would be a factor of a few smaller than the predicted turbulent Jeans length. However, taking potential projection effects into account, this difference may be less severe.

This turbulent Jeans fragmentation picture becomes questionable for IRDC 18310-4 if one considers that toward one of our most massive subcondensations (i.e., mmla with $6.6 M_{\odot}$ ) the measured line widths only show upper limits of $0.3 \mathrm{~km} \mathrm{~s}^{-1}$. Hence, no significant turbulent contribution to the line width is observed here.

A different approach to solving the discrepancies between the classical Jeans fragmentation and our data is to investigate the initial conditions, in particular the initial density structure. While the Jeans fragmentation analysis starts with uniform and infinite gas structures (e.g., Stahler \& Palla 2005), the derived density structures of the observed gas clumps exhibit much steeper density profiles (e.g., Beuther et al. 2002; Mueller et al. 2002; Hatchell \& van der Tak 2003). Girichidis et al. (2011) analyzed the different fragmentation properties during star formation in numerical simulations by varying the turbulent velocity fields as well as the density profiles from uniform to BonnorEbert sphere and density power-law profiles $\rho \propto r^{-p}$ with indices $p$ of 1.5 and 2. As expected, the steeper the initial density profile is, the less fragmentation they find and the more massive the final fragment masses are. In the extreme cases, the difference in masses rises by up to a factor of $\sim 20$. While they do not explicitly give the fragment separations, the fact that they find fewer fragments indicates that the fragment separation should also increase.

To summarize these effects, the observed fragmentation properties in IRDC 18310-4, as well as other sources in the literature, could most likely be explained by turbulence-suppressed fragmentation of gas clumps with non-uniform density structures. Magnetic fields can be another agent in inhibiting fragmentation (e.g., Commerçon et al. 2011; Pillai et al. 2015).

\subsection{Kinematic properties}

As outlined in Sect. 3.3, our new high spatial and high spectral resolution observations of the dense gas tracer $\mathrm{N}_{2} \mathrm{H}^{+}(3-2)$ resolve the spectral lines from the several cores into multiple spectral components where the individual components are only slightly above the thermal line width. Although the data are not yet good enough to actually resolve coherent thermally dominated structures like those found for example in B5 (Pineda et al. 2010, 2015), we are reaching a regime for the individual subcores within a high-mass star-forming region that is not that far from coherence.

However, in addition to the narrow line widths found for individual components, the omnipresence of multiple components toward each core implies additional highly dynamic processes within the overall high-mass star-forming region. The most straightforward interpretation of these multiple velocity components is within the picture of a globally collapsing gas clump where the different velocity components trace separate individual gas parcels that fall toward the gravitational center of the whole gas clump. The spectral line $\mathrm{N}_{2} \mathrm{H}^{+}(1-0)$ signatures of such a global collapse were simulated by Smith et al. (2013), and they find similar spectra with multiple components along individual lines of sight as seen in our IRDC 18310-4 data.

The $\mathrm{C}^{18} \mathrm{O}(2-1)$ data also allow us to derive a rough estimate of the virial mass. Following MacLaren et al. (1988) and assuming a density profile $\rho \propto 1 / r$, a radius of the clump of $0.25 \mathrm{pc}$ (Fig. 1), and the one-component fit $\Delta v \sim 2.0 \mathrm{~km} \mathrm{~s}^{-1}$ to the $\mathrm{C}^{18} \mathrm{O}(2-1)$ spectrum, the approximate virial mass is $\sim 190 M_{\odot}$, about a factor of 4 lower than the gas mass of $800 M_{\odot}$ derived from the dust continuum data (Beuther et al. 2013b) ${ }^{1}$.

In the framework of a globally collapsing gas clump, one can also use the observed spectral velocity differences for a simple collapse time estimate. Assuming that the difference between the velocity peaks is due to cores sitting at different points in a globally collapsing region, we get converging velocity differences along the lines of sight of $2.8 \mathrm{~km} \mathrm{~s}^{-1}$ in $\mathrm{mm} 1,5.2 \mathrm{~km} \mathrm{~s}^{-1}$ in $\mathrm{mm} 2$, and $1.8 \mathrm{~km}^{-1} \mathrm{~s}$ in $\mathrm{mm} 3$. Ignoring for this estimate the plane of the sky velocity component that we do not know, these velocity gradients would bring together fragments $10000 \mathrm{AU}$ apart in only $\sim 1 \times 10^{4}$ to $\sim 2.6 \times 10^{4} \mathrm{yr}$. The collapse of the clump may also cause the fragments to merge or increase in density, which could help explain the high masses of the fragments in the previous section. For example, Smith et al. (2009) found, using synthetic interferometry observations of simulated gas clumps, that the number of fragments decreased and their mean column density increased as the clumps collapsed. This could efficiently increase the mass of the fragments.

Combining the multiple components with the individual narrow line widths as well as the low virial mass, the most likely interpretation of the kinematic data in this region is a dynamical collapse of a large-scale gas clump that caused multiple velocity components along the line of sight, and where at the same time the individual infalling gas structures have very low levels of internal turbulence.

\section{Conclusions}

Resolving the mm continuum and $\mathrm{N}_{2} \mathrm{H}^{+}(3-2)$ emission at subarcsecond resolution (linear scales down to $\sim 2500 \mathrm{AU}$ ) toward the pristine high-mass starless gas clump IRDC 18310-4, we reveal the fragmentation and kinematic properties of the dense gas at the onset of massive star formation. Zooming through different size scales from single-dish data to intermediate and high

1 Density profiles steeper than $1 / r$ would result in even lower virial mass estimates. 
angular resolution PdBI observations, the resolved entities always fragment hierarchically into smaller substructures at the higher spatial resolution. While the fragment separations are still in approximate agreement with thermal Jeans fragmentation, the observed core masses are orders of magnitude larger than the estimated Jeans masses at the given densities and temperatures. Hence, additional processes have to be in place. However, taking into account non-uniform density structures as well as initial turbulent gas properties, observed core masses and projected separations are consistent with cloud formation models.

While most subcores are (far-)infrared dark even at $70 \mu \mathrm{m}$, the re-reduced Herschel data reveal weak $70 \mu \mathrm{m}$ emission toward core $\mathrm{mm} 2$ with a comparably low luminosity of only $\sim 16 L_{\odot}$. Since such a low luminosity cannot be caused either by an internal high-mass star or by strong accretion onto a typical embedded protostar, this supports the notion of the youth and early evolutionary stage of the region.

The spectral line data reveal multiple velocity components with comparably small widths (still above thermal) toward the individual subcores. Comparing these data to cloud collapse simulations, they agree with globally collapsing gas clumps where several gas parcels along the line of sight are revealed as individual spectral features. The narrow line widths in the 0.3 to $1 \mathrm{~km} \mathrm{~s}^{-1}$ regime indicate that even during the dynamical global collapse, individual subparcels of gas can have very low internal levels of turbulence.

\section{References}

Banerjee, R., Vázquez-Semadeni, E., Hennebelle, P., \& Klessen, R. S. 2009, MNRAS, 398, 1082

Bergin, E. A., Hartmann, L. W., Raymond, J. C., \& Ballesteros-Paredes, J. 2004, ApJ, 612, 921

Beuther, H., Schilke, P., Menten, K. M., et al. 2002, ApJ, 566, 945

Beuther, H., Churchwell, E. B., McKee, C. F., \& Tan, J. C. 2007, in Protostars and Planets V, eds. B. Reipurth, D. Jewitt, \& K. Keil, 165

Beuther, H., Linz, H., \& Henning, T. 2013a, A\&A, 558, A81

Beuther, H., Linz, H., Tackenberg, J., et al. 2013b, A\&A, 553, A115

Bontemps, S., Motte, F., Csengeri, T., \& Schneider, N. 2010, A\&A, 524, A18
Carey, S. J., Noriega-Crespo, A., Mizuno, D. R., et al. 2009, PASP, 121, 76 Commerçon, B., Hennebelle, P., \& Henning, T. 2011, ApJ, 742, L9

Csengeri, T., Urquhart, J. S., Schuller, F., et al. 2014, A\&A, 565, A75

Diolaiti, E., Bendinelli, O., Bonaccini, D., et al. 2000, A\&AS, 147, 335

Dobbs, C. L., Krumholz, M. R., Ballesteros-Paredes, J., et al. 2014, Protostars and Planets VI, 3

Draine, B. T. 2011, Physics of the Interstellar and Intergalactic Medium (Princeton Series in Astrophysics)

Girichidis, P., Federrath, C., Banerjee, R., \& Klessen, R. S. 2011, MNRAS, 413, 2741

Gutermuth, R. A., \& Heyer, M. 2015, AJ, 149, 64

Hatchell, J., \& van der Tak, F. F. S. 2003, A\&A, 409, 589

Heitsch, F., Hartmann, L. W., Slyz, A. D., Devriendt, J. E. G., \& Burkert, A. 2008, ApJ, 674, 316

Krumholz, M. R., Klein, R. I., \& McKee, C. F. 2007, ApJ, 656, 959

Linz, H., Krause, O., Beuther, H., et al. 2010, A\&A, 518, L123

MacLaren, I., Richardson, K. M., \& Wolfendale, A. W. 1988, ApJ, 333, 821

Motte, F., Bontemps, S., Schilke, P., et al. 2007, A\&A, 476, 1243

Motte, F., Nguyên Luong, Q., Schneider, N., et al. 2014, A\&A, 571, A32

Mueller, K. E., Shirley, Y. L., Evans, N. J., \& Jacobson, H. R. 2002, ApJS, 143, 469

Ossenkopf, V., \& Henning, T. 1994, A\&A, 291, 943

Pilbratt, G. L., Riedinger, J. R., Passvogel, T., et al. 2010, A\&A, 518, L1

Pillai, T., Kauffmann, J., Tan, J. C., et al. 2015, ApJ, 799, 74

Pineda, J. E., Goodman, A. A., Arce, H. G., et al. 2010, ApJ, 712, L116

Pineda, J. E., Offner, S. S. R., Parker, R. J., et al. 2015, Nature, 518, 213

Poglitsch, A., Waelkens, C., Geis, N., et al. 2010, A\&A, 518, L2

Ragan, S., Henning, T., Krause, O., et al. 2012, A\&A, 547, A49

Ragan, S. E., Henning, T., Beuther, H., Linz, H., \& Zahorecz, S. 2015, A\&A, 573, A119

Reid, M. J., Schneps, M. H., Moran, J. M., et al. 1988, ApJ, 330, 809

Roussel, H. 2013, PASP, 125, 1126

Russeil, D., Zavagno, A., Motte, F., et al. 2010, A\&A, 515, A55

Sánchez-Portal, M., Marston, A., Altieri, B., et al. 2014, Exp. Astron., 37, 453

Smith, R. J., Clark, P. C., \& Bonnell, I. A. 2009, MNRAS, 396, 830

Smith, R. J., Shetty, R., Beuther, H., Klessen, R. S., \& Bonnell, I. A. 2013, ApJ, 771,24

Stahler, S. W., \& Palla, F. 2005, The Formation of Stars (Wiley-VCH)

Tackenberg, J., Beuther, H., Henning, T., et al. 2012, A\&A, 540, A113

Tan, J. C., Beltrán, M. T., Caselli, P., et al. 2014, Protostars and Planets VI, 149

Vázquez-Semadeni, E., Ryu, D., Passot, T., González, R. F., \& Gazol, A. 2006, ApJ, 643, 245

Wang, K., Zhang, Q., Testi, L., et al. 2014, MNRAS, 439, 3275

Zhang, Q., Wang, K., Lu, X., \& Jiménez-Serra, I. 2015, ApJ, 804, 141

Zinnecker, H., \& Yorke, H. W. 2007, ARA\&A, 45, 481 